\title{
EGFR or HER2 inhibition modulates the tumor microenvironment by suppression of PD-L1 and cytokines release
}

\author{
Koung Jin Suh ${ }^{1,3, *}$, Ji Hea Sung ${ }^{1, *}$, Jin Won Kim ${ }^{1}$, Song-Hee Han ${ }^{2}$, Hye Seung Lee ${ }^{2}$, \\ Ahrum Min', Mi Hyun Kang ${ }^{1}$, Ji Eun Kim ${ }^{1}$, Ji-Won Kim ${ }^{1}$, Se Hyun Kim${ }^{1}$, Jeong-OK \\ Lee $^{1}$, Yu Jung Kim${ }^{1}$, Keun-Wook Lee ${ }^{1}$, Jee Hyun Kim ${ }^{1}$, Soo-Mee Bang ${ }^{1}$, Seock-Ah \\ Im $^{3,4}$ and Jong Seok Lee ${ }^{1}$ \\ ${ }^{1}$ Department of Internal Medicine, Seoul National University Bundang Hospital, Seoul National University College of Medicine, \\ Bundang-gu, Seongnam, Korea \\ ${ }^{2}$ Department of Pathology, Seoul National University Bundang Hospital, Seoul National University College of Medicine, \\ Bundang-gu, Seongnam, Korea \\ ${ }^{3}$ Department of Internal Medicine, Seoul National University Hospital, Seoul National University College of Medicine, \\ Jongno-gu, Seoul, Korea \\ ${ }^{4}$ Cancer Research Institute, Seoul National University, Jongno-gu, Seoul, Korea \\ *These authors have contributed equally to this work \\ Correspondance to: Jin Won Kim, email: jwkim@snubh.org \\ Keywords: PD-L 1, cytokine, EGFR, HER2, PI3K \\ Received: January 04, $2017 \quad$ Accepted: June 11,2017 Published: July 12, 2017 \\ Copyright: Suh et al. This is an open-access article distributed under the terms of the Creative Commons Attribution License 3.0 \\ (CC BY 3.0), which permits unrestricted use, distribution, and reproduction in any medium, provided the original author and source \\ are credited.
}

\section{ABSTRACT}

Background: Characteristics of tumor microenvironment have been suggested as predictive markers of anti-EGFR or anti-HER2 treatment response. However, the effect of EGFR/HER2 signal blockade on the tumor immune microenvironment is unclear.

Methods: EGFR/HER2 pathway signaling and PD-L1 expression in gastric cancer cell lines were screened by western blot analysis. PD-L1 and HER2 expressions in 251 resected gastric tumors were determined by immunohistochemistry, and changes in EFGR, HER2, and PD-L1 expression in paired specimens between preand post-chemotherapy were evaluated. PD-L1 expression in HER2-amplified cell lines was evaluated by western blotting, fluorescence-activated cell sorting, reverse transcription, and real-time quantitative PCR analyses before and after afatinib, lapatinib, pictilisib and trametinib treatment. Changes in cytokines were evaluated by reverse transcription, real-time quantitative PCR, and enzyme-linked immunosorbent assay after EGFR/HER2 inhibition.

Results: Cell lines with PEGFR or pHER2 overexpression showed higher PD-L1 expression. In resected gastric tumors, HER2 expression was significantly associated with PD-L1 expression $(p=0.030)$. PD-L1 overexpression accompanied by increased HER2 expression was identified in a post-chemotherapy specimen from a patient with an initial HER2/PD-L1-negative tumor. In HER2-overexpressing cell lines, PD-L1 expression was decreased in a dose- and time-dependent manner after afatinib and lapatinib treatment. PI3K pathway inhibition by pictilisib, but not MEK pathway inhibition by trametinib, resulted in PD-L1 suppression. After lapatinib treatment, the release of CCL2, CCL21, VEGF and CXCL1 decreased in a dose-dependent manner.

Conclusions: Inhibition of the EGFR/HER2 signaling pathway, particularly of downstream PI3K activity, suppressed PD-L1 and release of cytokines, suggesting that EGFR/HER2 inhibition may create a more favorable milieu for tumor immunotherapy. 


\section{INTRODUCTION}

The interaction between tumors and the tumor microenvironment is complicated. Tumor cells and the components of the tumor microenvironment communicate directly through cell-to-cell contact and indirectly through paracrine signals, which predominantly involve cytokines $[1,2]$. The programmed cell death-ligand 1 (PD-L1) on tumor cells has an important role in avoiding host immune surveillance by interacting with the programmed cell death-1 (PD-1) on immune cells in the tumor microenvironment $[3,4]$. Cytokines that are secreted by the tumor cells or by the normal cells recruited to the tumor microenvironment can function as growth factors, increase metastasis formation and angiogenesis, and induce the formation of an immunosuppressive microenvironment $[5,6]$.

Targeting the PD-1/PD-L1 immune checkpoint signaling to restore cancer cell-directed immune response is a confirmed strategy for several tumor types. Although there are controversies, PD-L1 expression has been suggested as a predictive marker of PD-1/PD-L1 checkpoint inhibitor treatment response [7-9]. Recent studies have suggested that the predictive value of PDL1 overexpression might not be restricted to immune checkpoint inhibitor treatments. D'Incecco et al. reported that in patients with non-small cell lung cancer (NSCLC) treated with epidermal growth factor receptor (EGFR) tyrosine kinase inhibitors (TKIs), the response rate, time to progression, and overall survival (OS) were significantly better in PD-L1-positive tumors [10]. High PD-L1 expression was positively associated with EGFR mutation status [11] and better EGFR TKI treatment outcome in patients with EGFR-mutant NSCLC [12]. In addition, higher levels of tumor-infiltrating lymphocytes (TILs), another important participant in antitumor immunity, were significantly associated with decreased distant recurrence in patients with HER2positive breast cancer treated with the anti-HER2 monoclonal antibody, trastuzumab [13]. Taken together, these data indicate that overexpression of PD-L1 is associated with EGFR/HER2 status, and drugs targeting these pathways may relieve suppression of antitumor effector immunity. However, the mechanism by which oncogenic signaling modulates tumor immunity in the tumor microenvironment has not been elucidated. There are only a few studies that have addressed this issue by investigating the oncogenic activation of the AKTmTOR pathway [14, 15] or the MEK-ERK pathway [15] and NF-Kb regulation [16].

To elucidate the effect of EGFR/HER2 signal blockade on the tumor immune microenvironment, we examined the association between PD-L1 expression and the EGFR/HER2 signaling pathway and the direct effect of EGFR/HER2 pathway inhibition on the cytokines release by tumor cells.

\section{RESULTS}

\section{Association between PD-L1 and EGFR/HER2 signaling in gastric cancer cell lines}

To determine the association between PD-L1 and the EGFR/HER2 pathway, we examined the protein expressions of PD-L1 and molecules related to the EGFR/ HER2 signaling pathway in seven gastric cancer cell lines. Western blot analysis revealed that SNU216, SNU668, and NCI-N87 had higher p-EGFR, p-HER2, and PD-L1 expression (Figure 1). These results suggest a positive association between PD-L1 expression and EGFR/ HER2 signaling pathway activation. This finding was also confirmed in HER2-amplified breast cancer cell line (SKBR3) and EGFR-mutated lung cancer cell line (PC9) as positive controls.

To determine the clinical relevance of the findings, we evaluated PD-L1 and HER2 expressions in 251 surgically resected gastric tumor tissues from our previously reported gastric cancer cohort [17]. PD-L1 and HER2 protein expressions $(1+, 2+, 3+)$ in $173(68.9 \%)$ and $68(27.1 \%)$ patients, respectively, were evaluated. Among the patients with negative HER2 protein expression $(\mathrm{n}=$ $183), 63.4 \%$ had positive PD-L1 expression $(\mathrm{n}=116)$. PD-L1 expression was more frequent in patients with positive HER2 protein expression; $89.5 \%$ (34/38), 75.0\% $(12 / 16)$, and $78.6 \%(11 / 14)$ of the patients with $1+, 2+$, and 3+ HER2 expression, respectively, had PD-L1-positive tumors, thus demonstrating a significant linear association $(p=0.030)$ (Table 1).

The EGFR, HER2, and PD-L1 expressions in paired specimens of tumors obtained pre- and post-chemotherapy were evaluated by immunohistochemistry (IHC). A total of 10 specimens from five patients were included in this analysis. In one patient with an initial HER2-negative tumor, the post-chemotherapy specimen showed increased PD-L1 and HER2 expression levels (Figure 2). In the other four patients, the expression levels of EGFR, HER2, and PD-L1 did not show any difference between pre- and post-chemotherapy.

\section{Changes in PD-L1 expression following EGFR/ HER2 signaling blockade in HER2-amplified cell lines}

To determine the effect of EGFR/HER2 signaling pathway inhibition on PD-L1 expression, the changes in PD-L1 expression following EGFR/HER2 blockade in HER2-amplified cell lines (SNU216, NCI-N87, and SKBR3) were evaluated using afatinib and lapatinib (dual kinase inhibitor of EGFR and HER2). The protein expression of PD-L1 was suppressed in a dose-dependent manner when pHER2 was suppressed by lapatinib and afatinib (Figure 3A and 3B). The mRNA expression of PD-L1 was also reduced in a dose-dependent manner, as 
Table 1: Association between PD-L1 expression status and clinicopathological characteristics

\begin{tabular}{lcccc}
\hline \multirow{2}{*}{ Variables } & \multicolumn{2}{c}{ PD-L1 } & & Total 251 \\
\cline { 2 - 4 } & Positive (173) & Negative (78) & & P-value \\
\hline HER2 IHC Negative & $116(63.4 \%)$ & $67(36.6 \%)$ & 183 & 0.030 \\
HER2 IHC 1+ & $34(89.5 \%)$ & $4(10.5 \%)$ & 38 \\
HER2 IHC 2+ & $12(75.0 \%)$ & $4(25.0 \%)$ & 16 \\
HER2 IHC 3+ & $11(78.6 \%)$ & $3(21.4 \%)$ & 14 \\
\hline
\end{tabular}

IHC immunohistochemistry. HER2 immunohistochemistry results are scored as IHC 0 (negative), IHC 1+ (negative), IHC $2+$ (equivocal) or IHC 3+ (positive).

determined by reverse transcription PCR (RT-PCR) and real-time quantitative PCR (qPCR) (Figure 3C and 3D). The expression of PD-L1 was decreased with increasing lapatinib treatment, as assessed by flow cytometer (Figure $3 \mathrm{E})$. Moreover, when the cell lines were treated with 0.1 $\mu \mathrm{M}$ lapatinib for different times $(0,4,24$, and $72 \mathrm{~h})$, a gradual pattern of PD-L1 suppression was observed (Figure 3F). These data indicate that EGFR/HER2 blockade could reduce the expression of PD-L1 in HER2amplified cancer cells.

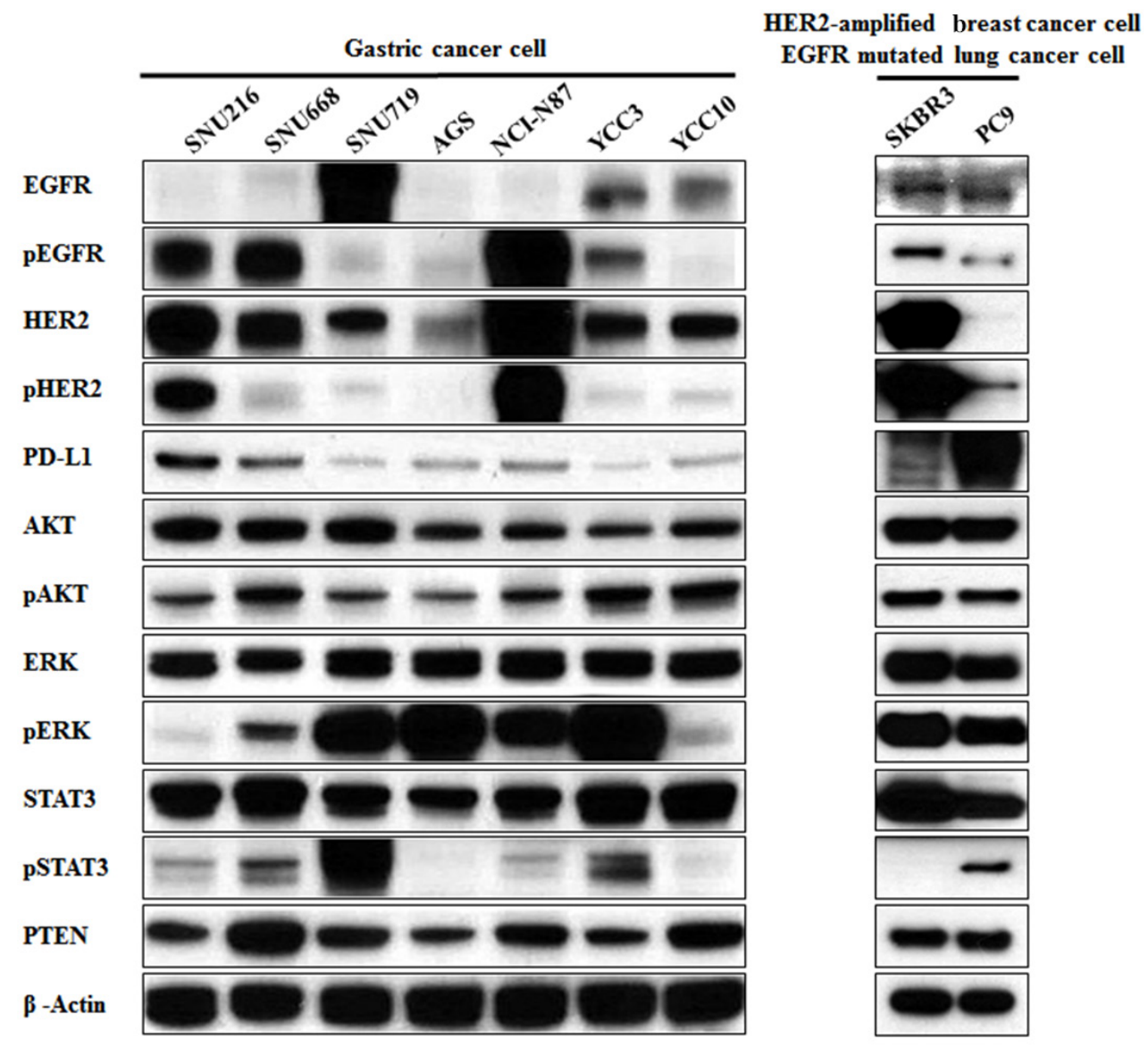

Figure 1: Association of PD-L1 expression with the EGFR/HER2 signaling pathway in gastric cancer cell lines. Seven gastric cancer cell lines (SNU216, SNU668, SNU719, AGS, NCI-N87, YCC3 and YCC10) were seeded at a density of $1 \times 10^{6}$ cells per well in 6-well plates and incubated in a humidified $\mathrm{CO}_{2}$ incubator at $37^{\circ} \mathrm{C}$. The protein expressions of molecules related to the EGFR/ HER2 signaling pathway were screened by western blot analysis. PD-L1 expression in cell lines with pEGFR overexpression or pHER2 overexpression (SNU216, SNU668, and NCI-N87) was relatively higher than that in other cell lines. As positive controls, HER2-amplified breast cancer cell line (SKBR3) and EGFR-mutated lung cancer cell line (PC9) showed higher PD-L1 expression. 


\section{PD-L1 suppression via inhibition of AKT signaling in HER2-amplified cell lines}

The PI3K-AKT-mTOR and RAS-RAF-MEK pathways are two of the most important downstream pathways of EGFR/HER2 signaling. We hypothesized that one of these downstream pathways could be dominantly involved in the regulation of PD-L1 by HER2 overexpression. To determine whether PD-L1 expression was dependent on active PI3K-AKT-mTOR signaling or RAS-RAF-MEK signaling, HER2-amplified cell lines with high PD-L1 expression were treated with pharmacologic inhibitors of specific components in the pathway. We treated the SNU216, NCI-N87, and SKBR3 cell lines with $0.1 \mu \mathrm{M}$ pictilisib (a selective inhibitor of class I PI3Ks), $0.1 \mu \mathrm{M}$ trametinib (a MEK inhibitor), $0.1 \mu \mathrm{M}$ lapatinib, and $0.02 \mu \mathrm{M}$ afatinib. By using flow cytometer, we found that the expression of PD-L1 was reduced in pictilisibtreated, lapatinib-treated, and afatinib-treated cell lines (Figure 4A). In contrast, the expression of PD-L1 was not suppressed in the trametinib-treated SNU216 and SKBR3 cell lines.

To more clearly demonstrate the effect of inhibition, cell lines were treated with interferon gamma (IFN $\gamma$ ) to induce PD-L1 expression. Indeed, PD-L1 expression in the cell lines was stimulated after IFN $\gamma$ treatment. Pictilisib but not trametinib inhibited the induction of PD-L1 through IFN $\gamma$ both at the protein and mRNA level (Figure 4B and 4C). These results suggest the association of ERGF/HER2 with PD-L1 via the PI3K-AKT-mTOR pathway dominantly.

\section{Changes in cytokines release following EGFR/ HER2 blockade}

We examined the direct effect of EGFR/HER2 inhibition on the cytokines release of tumor cell lines. SNU216, NCI-N87, and SKBR3 cell lines were treated with different concentrations of lapatinib $(0,0.01,0.1$, and $1 \mu \mathrm{M})$. The mRNA expression of CCL2, CCL21, and CXCL1 was suppressed in a dose-dependent manner (Figure 5A and 5B). Furthermore, in enzyme-linked immunosorbent assay (ELISA) of supernatants after $24 \mathrm{~h}$ treatment with lapatinib, the concentration of CCL2, CCL21, VEGF and CXCL1 were decreased in a dosedependent manner, although dramatic decrease of CCL2 concentration did not be shown due to very low baseline concentration in SNU216 and NCI-N87.

\section{DISCUSSION}

In the present study, we demonstrated that PD-L1 expression was higher in gastric cancer cell lines with higher pEGFR and pHER2 expressions compared with those with lower pEGFR and pHER2 expressions, and inhibition of the HER2/EGFR pathway decreased PD-L1 expression as well as cytokines release in HER2-amplified cancer cell lines. Furthermore, inhibition of the PI3K but not MEK pathway reduced PD-L1 expression in these cell lines, thus suggesting that the modulation of PD-L1 expression by the EGFR/HER2 pathway is likely to be mediated via downstream PI3K-AKT-mTOR signaling.

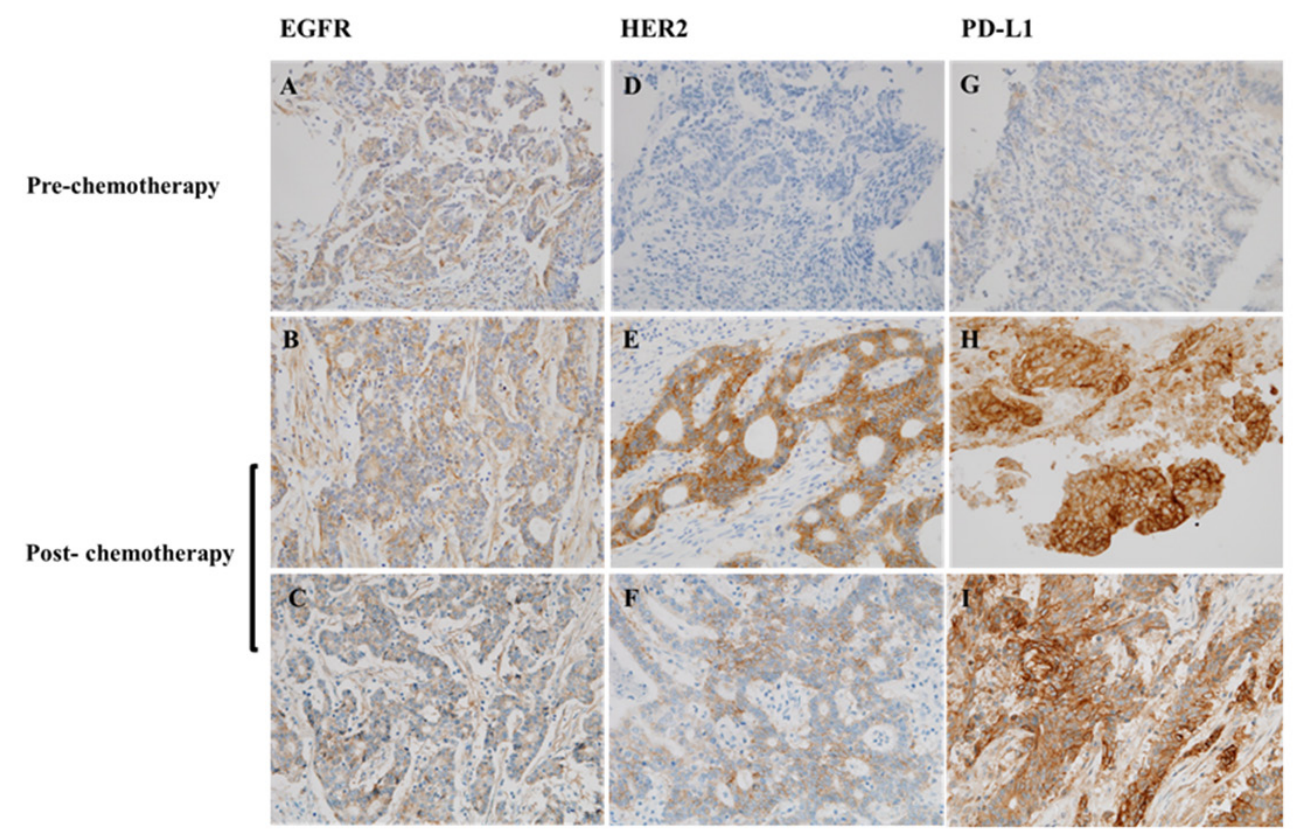

Figure 2: Immunohistochemistry analysis of the paired tumor specimens of pre-chemotherapy and post-chemotherapy. The specimens were stained for (A-C) EGFR, (D-F) HER2, and (G-I) PD-L1 expression. HER2 and PD-L1 were overexpressed in the post-chemotherapy specimen of an initial HER2/PD-L1-negative tumor; original magnification $\times 400$. 
The interaction between tumors and the tumor microenvironment is complicated. Signaling pathways of the inner tumor can be associated with the tumor microenvironment of the outer tumor. The tumor microenvironment also influences tumor behavior. PDL1 is a transmembrane surface glycoprotein expressed by lymphocytes and dendritic cells as well as aberrantly on the surface of epithelial cells in a wide range of solid tumors. In addition, PD-L1 is a ligand of PD-1, which is known to be expressed by activated T-cells in the germinal centers of lymphoid follicles as well as TILs [3]. The interaction between PD-1 and its ligand PD-L1 suppresses T-cell receptor signaling and results in immune system downregulation, which in turn enables cancer cells to escape immune destruction [4]. Several cytokines, such as CCL2, CCL21, VEGF and CXCL1 are present
$\mathbf{A}$

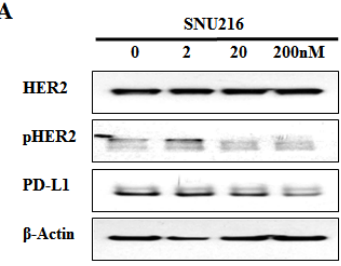

B

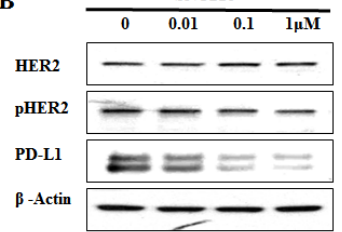

C

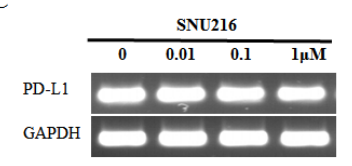

D

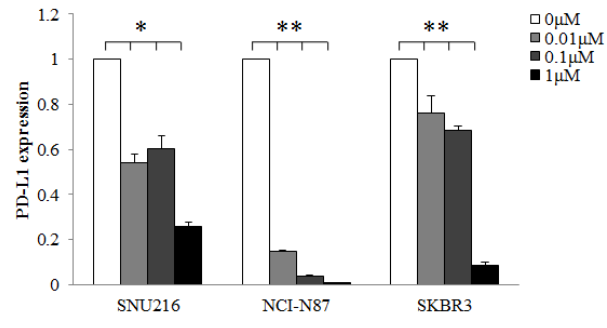

$\mathbf{F}$
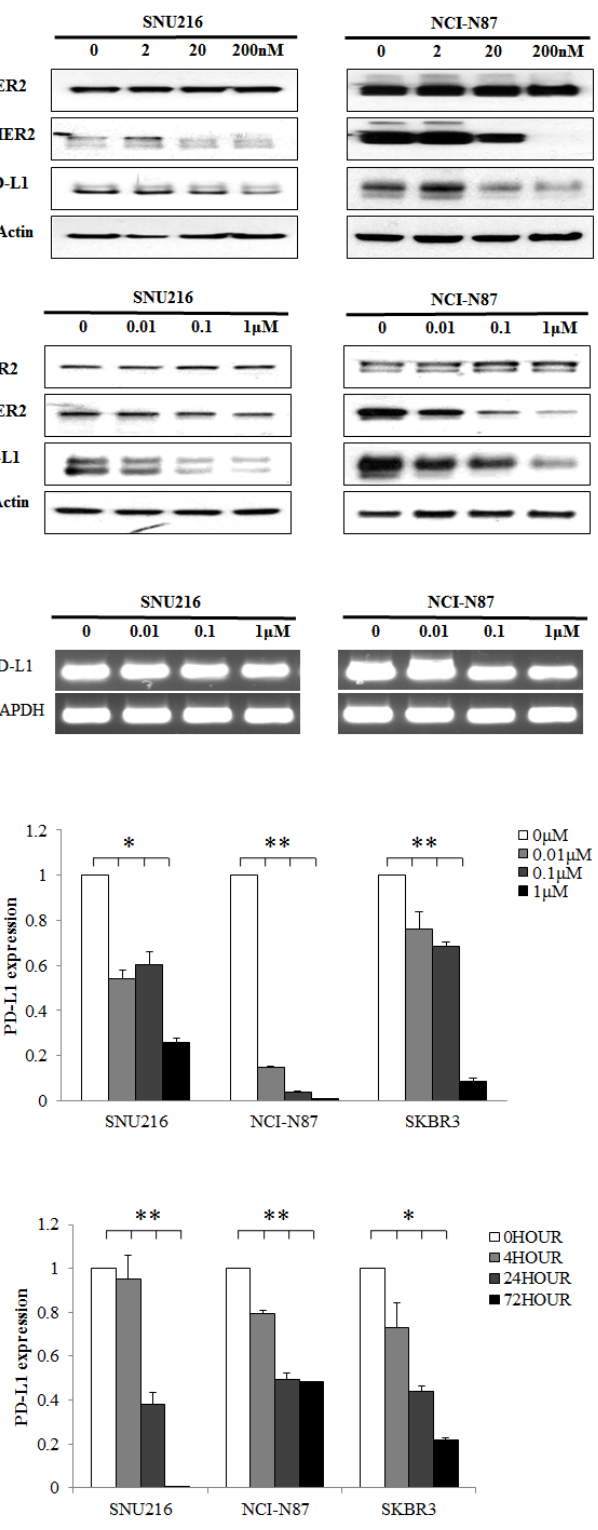
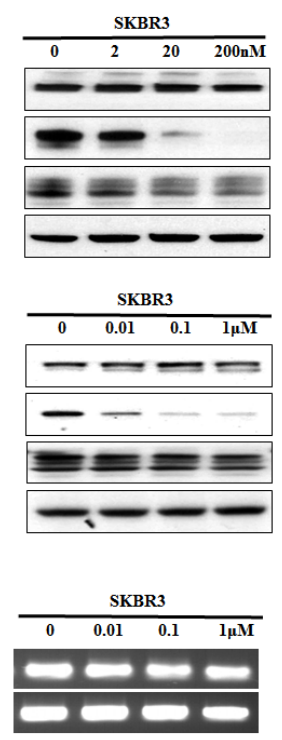

$\mathbf{E}$
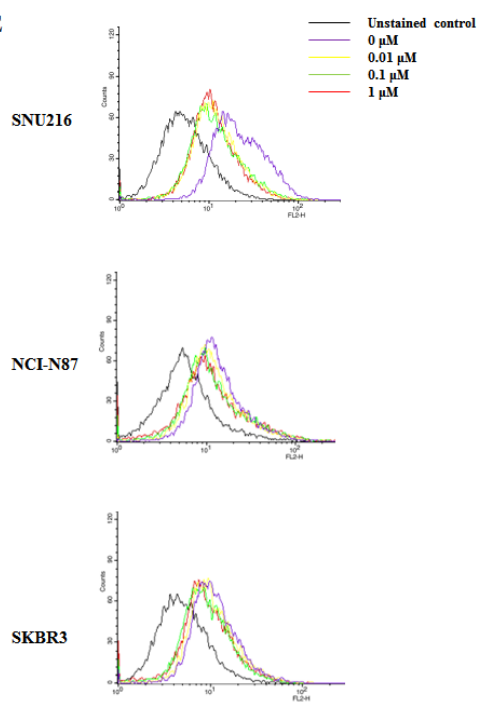
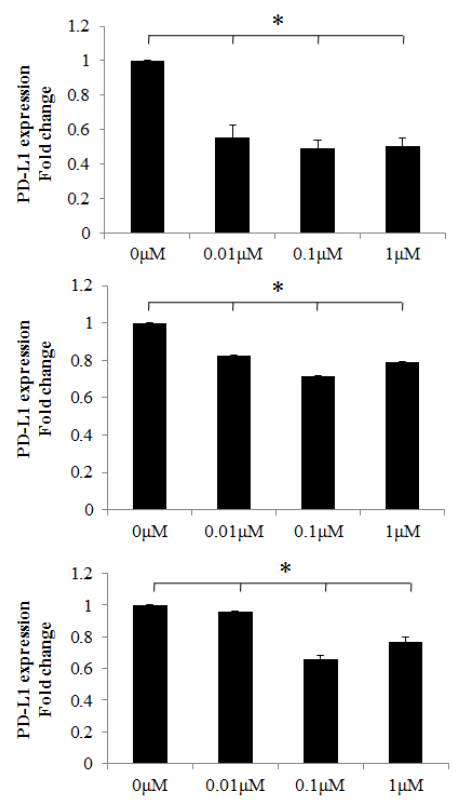

Figure 3: Decrease in PD-L1 expression following EGFR/HER2 inhibition in HER2-amplified cell lines. HER2-amplified gastric (SNU216, NCI-N87) and breast (SKBR3) cancer cell lines were seeded at a density of $1 \times 10^{6}$ cells per well in 6-well plates and incubated in a humidified $\mathrm{CO}_{2}$ incubator at $37^{\circ} \mathrm{C}$. After overnight growth, (A) afatinib $(0,2,20$, and $200 \mathrm{nM})$ and (B) lapatinib $(0,0.01$, 0.1 , and $1 \mu \mathrm{M}$ ) were added. Cells were harvested at $24 \mathrm{~h}$, and the expression of PD-L1 was analyzed by western blot analysis. PD-L1 was suppressed in a dose-dependent manner. The mRNA expression of PD-L1 after lapatinib $(0,0.01,0.1$, and $1 \mu \mathrm{M})$ treatment was determined by (C) RT-PCR and (D) qPCR. (E) The mean fluorescence intensity of PD-L1 was determined by flow cytometry after lapatinib $(0,0.01$, 0.1 , and $1 \mu \mathrm{M})$ treatment. (F) PD-L1 expression was examined by qPCR after treatment with $0.1 \mu \mathrm{M}$ lapatinib for different times $(0,4,24$, and $72 \mathrm{~h}$ ). The PD-L1 expression of three independent experiments is shown as mean $\pm \mathrm{SD} . * 0.001 \leq p<0.05$ and $* * p<0.001$ in ANOVA. 
at high levels in the tumor microenvironment and known to be involved in tumor growth promotion, vascular development, and evasion of antitumor immunity. These molecules are thought necessary for tumors to interact with their microenvironment. In this study, decrease of cytokines release after lapatinib treatment was reported, which might give an indirect evidence for tumor treatment itself to modulate tumor microenvironment.

Recent studies have demonstrated that PD-L1 expression is not only a marker of response to immune
A

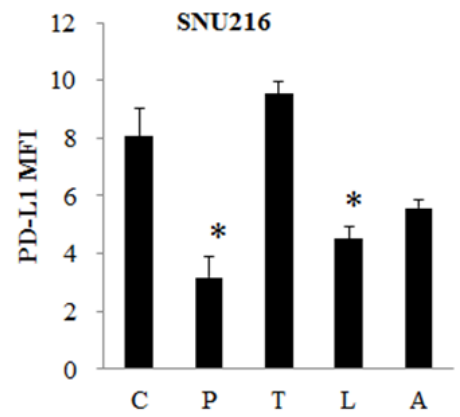

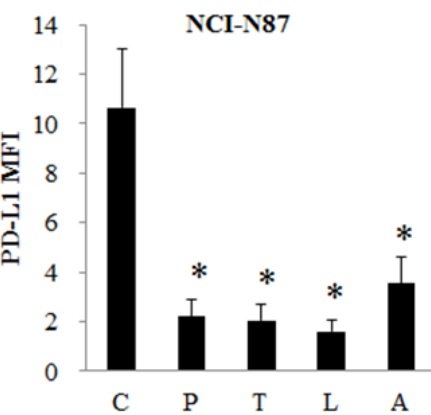

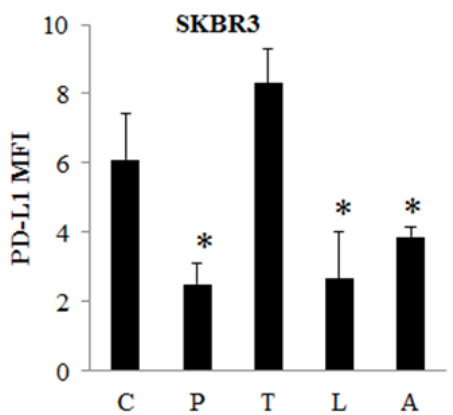

C. Control, P. Pictilisib $0.1 \mu \mathrm{M}, \mathrm{T}$. Trametinib $0.1 \mu \mathrm{M}, \mathrm{L}$. Lapatinib $0.1 \mu \mathrm{M}, \mathrm{A}$. Afatinib $0.02 \mu \mathrm{M}$

B

PD-L1

AKT

pAKT

ERK

pERK

$\beta$-Actin
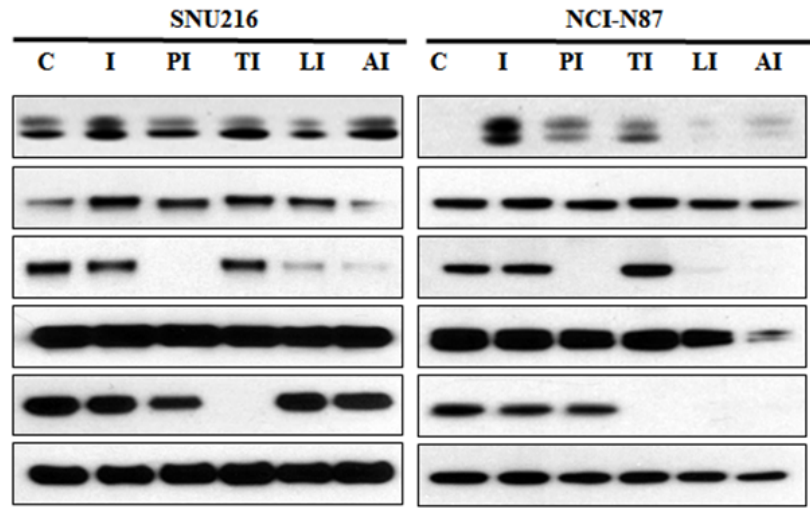

C. Control, I. Interferon gamma (IFN $\gamma$ ), PI. Pictilisib $1 \mu \mathrm{M} / \mathrm{IFN} \gamma$, TI. Trametinib $1 \mu \mathrm{M} / \mathrm{FN} \gamma$, LI. Lapatinib $1 \mu \mathrm{M} / \mathrm{IFN} \gamma$, AI. Afatinib $0.2 \mu \mathrm{M} / \mathrm{FN} \gamma$

C
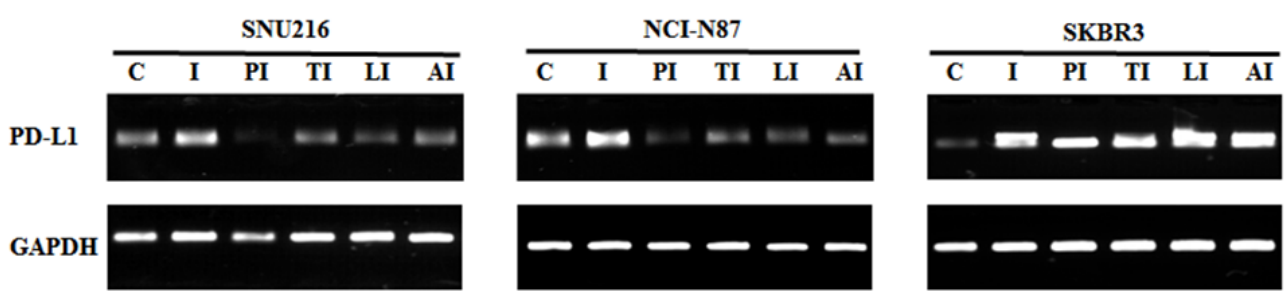

C. Control, I. Interferon gamma (IFN $\gamma$ ), PI. Pictilisib $1 \mu \mathrm{M} / \mathrm{IFN} \gamma$, TI. Trametinib $1 \mu \mathrm{M} / \mathrm{FN} \gamma$, LI. Lapatinib $1 \mu \mathrm{M} / \mathrm{FN} \gamma$, AI. Afatinib $0.2 \mu \mathrm{M} / \mathrm{FN} \gamma$

Figure 4: Involvement of the AKT pathway in PD-L1 expression regulation. HER2-amplified cancer cell lines were seeded at a density of $1 \times 10^{6}$ cells per well in 6-well plates and incubated in a humidified $\mathrm{CO}_{2}$ incubator at $37^{\circ} \mathrm{C}$. After overnight growth, cells were treated with $0.1 \mu \mathrm{M}$ pictilisib, $0.1 \mu \mathrm{M}$ trametinib, $0.1 \mu \mathrm{M}$ lapatinib, and $0.02 \mu \mathrm{M}$ afatinib for $24 \mathrm{~h}$. (A) The mean fluorescence intensity (MFI) of PD-L1 was determined by flow cytometry. PD-L1 was expression decreased in pictilisib-treated cell lines. In contrast, PD-L1 expression was not suppressed in trametinib-treated SNU216 and SKBR3 cell lines. To enhance PD-L1 expression, IFN $\gamma$ (10 $\mathrm{ng} / \mathrm{mL}$ ) was added after $4 \mathrm{~h}$ of drug treatment, and incubation was continued for a further $24 \mathrm{~h}$. At $28 \mathrm{~h}$ after drug treatment, cells were harvested for evaluation of PD-L1 protein expression by (B) western blotting and (C) RT-PCR analyses. In comparison with trametinib inhibition, pictilisib inhibition of the AKT pathway suppressed IFN $\gamma$-mediated PD-L1 protein upregulation. The PD-L1 expression of three independent experiments is shown as mean $\pm \mathrm{SD} .{ }^{*} p<0.05$ in paired Student's t-test (2-tailed). 
checkpoint inhibitor treatment but also cytotoxic chemotherapy and targeted therapy. PD-L1 expression can predict pathologic complete response in neoadjuvant chemotherapy-treated patients with breast cancer [19]. A study in patients with EGFR-mutant NSCLC following EGFR TKI therapy revealed that the outcome of patients with PD-L1-positive tumors was better compared with those with PD-L1-negative tumors [20]. Furthermore, another study found significantly greater levels of PDL1 through EGFR pathway activation after acquiring resistance to gefitinib in NSCLC cell lines [21]. An increase in PD-L1-positive peripheral blood T-cells after EGFR TKI treatment has been strongly associated with disease progression, thus suggesting that PD-L1 upregulation might also be one of the mechanisms of acquired resistance to EGFR TKIs [22]. Therefore, we suggest that overexpression of PD-L1 is associated with the EGFR/HER2 pathway, and the drugs targeting these pathways may relieve suppression of antitumor effector immunity. Since prognosis and treatment response of agents targeting the growth signaling pathways of the inner tumor are different depending on the tumor microenvironment, it is important to evaluate the effect of tumor oncogenic signaling on the tumor microenvironment. We found that the PI3K pathway was associated with PD-L1 expression. Similar to the present study, Lastwika et al. recently reported a strong association between PD-L1 protein expression and activation of the AKT-mTOR pathway in lung cancer [14]. They found that inhibition of PI3K, AKT, or mTOR decreased PDL1 expression in EGFR-mutated NSCLC cell lines, which was similar to our finding in HER2-amplified cancer cell lines. Ota et al. also reported that expression of PDL1 was suppressed by inhibitors of the MEK-ERK and PI3K-AKT signaling pathways in NSCLC cells positive for either EML4-ALK or activating mutations of the EGFR [15]. These reports including our result reveal an indirect link between tumor oncogenic signaling and tumor microenvironment.

In our tissue microarray data, HER2 was expressed in approximately $33 \%$ of tumors with PD-L1 expression, and PD-L1 was expressed in $84 \%$ of tumors with HER2 protein expression. HER2 amplification was not associated with PD-L1 expression in our cohort, which is in contrast with a study reporting HER2 overexpression in nearly $50 \%$ of PD-L1-positive gastric cancer cases [23]. According to TCGA data, which divided gastric cancer into four distinct subtypes [24], PD-L1/L2 expression is elevated in EBV-positive tumors; in contrast, HER2 amplification is most frequently found in chromosomal instability subtypes. These data along with our findings suggest that PD-L1 status is not always associated with HER2 amplification. However, one paired specimen demonstrated increased PD-L1 and HER2 expression after
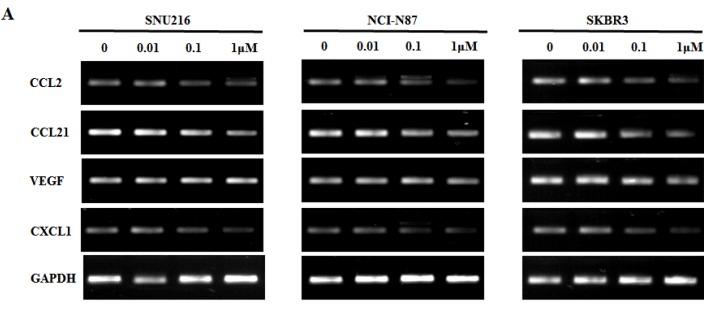

B
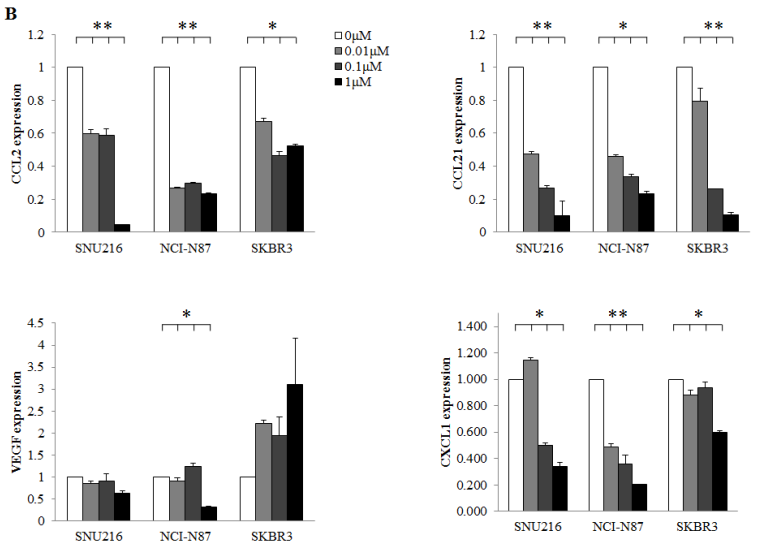
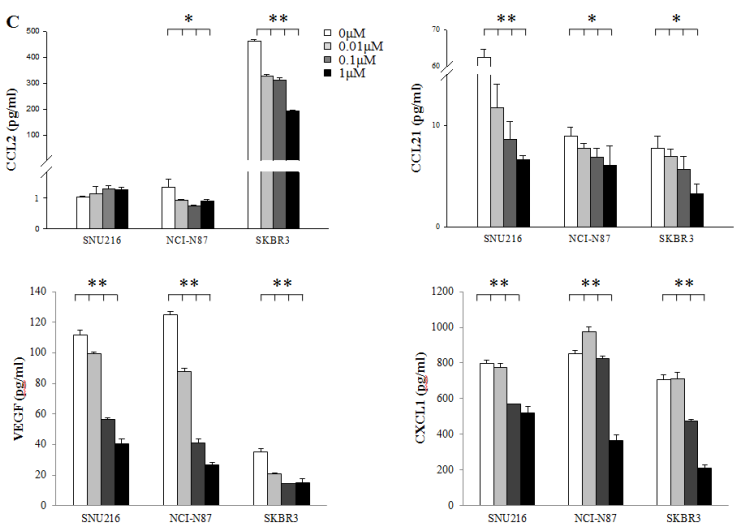

Figure 5: Effect of HER2 blockade on the cytokine release of tumor cells. HER2-amplified cell lines were seeded at a density of $1 \times 10^{6}$ cells per well in 6-well plates and incubated in a humidified $\mathrm{CO}_{2}$ incubator at $37^{\circ} \mathrm{C}$. After overnight growth, lapatinib $(0,0.01,0.1$, and $1 \mu \mathrm{M}$ ) was added. Cells were harvested after $24 \mathrm{~h}$. The mRNA expression of CCL2, CCL21, VEGF, and CXCL1 was examined by (A) RT-PCR, (B) qPCR, and (C) ELISA which demonstrated a dose-dependent suppression. The expression of three independent experiments is shown as mean $\pm \mathrm{SD}$. ${ }^{*} 0.001 \leq p<0.05$ and $* * p<0.001$ in ANOVA. 
chemotherapy in the present study. Although PD-L1 status might not be associated with HER2 expression, PD-L1 expression could be activated or suppressed by the EGFR/ HER2 pathway.

Our study has several limitations. The effect of EGFR/HER2 inhibition on PD-L1 and cytokine release was only evaluated in pEGFR and/or pHER2 overexpressed cell lines; thus, a possible association between the EGFR/HER2 pathway and PD-L1 expression in other cell lines could have been overlooked. To address the microenvironment impact of the treatment, 2-dimensional experiments have limitation. Although coculture system with immune cells may be better to prove the concepts, this study could not incorporate this system. In addition, we were unable to evaluate the change in PD-L1 expression and cytokines release in tumor tissues obtained from patients treated with different inhibitors used in this study (PI3K inhibitor and MEK inhibitor) since these agents are not used in routine clinical practice. Nevertheless, paired tissues of pre-chemotherapy and post-chemotherapy were available and the association between PD-L1 and EGFR/HER2 was evaluated. Further in vivo studies are warranted to confirm the findings in our in vitro study.

Overall, our findings suggest that agents targeting EGFR/HER2 could inhibit the induction of PD-L1, which may be dependent on the PI3K-AKT-mTOR pathway and suppress cytokines release in HER2-amplified cancer cells. EGFR/HER2 inhibition may create a more favorable milieu for tumor immunotherapy.

\section{MATERIALS AND METHODS}

\section{Cell lines and reagents}

NCI-N87, AGS, and SKBR3 cells were obtained from the American Type Culture Collection. SNU216, SNU668, and SNU719 cells were obtained from the Korean Cell Line Bank. YCC3, YCC10, and PC9 cells were obtained from Cancer Metastasis Research Center of Yonsei University College of Medicine. All cell lines were authenticated using by short tandem repeats. All were cultured in RPMI 1640 (Gibco, grand Island, NY) with L-glutamine (300 mg/L), supplemented with $25 \mathrm{mM}$ HEPES, $25 \mathrm{mM} \mathrm{NAHCO}$, $10 \%$ fetal bovine serum (FBS; Gibco, grand Island, NY), penicillin $(100 \mathrm{U} / \mathrm{mL})$, and streptomycin $(50 \mu \mathrm{g} / \mathrm{mL}$, WelGENE Inc, Daegu, Korea). The cell cultures were maintained in a humidified $\mathrm{CO}_{2}$ incubator at $37^{\circ} \mathrm{C}$. The drugs including Afatinib, pictilisib, trametinib, and lapatinib were purchased from Selleck Chemicals (Houston, TX, USA). IFN $\gamma$ was obtained from ProSpec-Tany TechnoGene Ltd. (Rehovot, Israe). SNU216, NCI-N87, and SKBR3 were seeded in 6-well tissue culture plates at a density of $1 \times 10^{6}$ cells per well. Cells were cultured in RPMI 1640 with $10 \%$ fetal bovine serum. After overnight growth, the cells were treated with specific drugs at different concentrations and time points. After each exposure time of drugs, cells were harvested for experiments. To enhance PD-L1 expression, IFN $\gamma$-induction was applied. After cell seeding and overnight growth, the cells were treated with specific drugs. And then, IFN $\gamma(10 \mathrm{ng} / \mathrm{mL})$ was added after $4 \mathrm{~h}$ of drug treatment, and incubation was continued for a further $24 \mathrm{~h}$. At $28 \mathrm{~h}$ after drug treatment, cells were harvested for experiments.

\section{Western blot analysis}

Cells were lysed in $1 \times$ lysis buffer (Cell Signaling Technology, Danvers, MA, USA) containing $1 \mathrm{mM}$ of the protease inhibitor PMSF (Amresco, Solon, OH, USA), and the cell extracts were centrifuged at $16000 \mathrm{~g}$ for 15 min. All samples were denatured in buffer containing $60 \mathrm{mM}$ Tris, pH 6.8, 25\% glycerol, 2\% SDS, $14.4 \mathrm{mM}$ 2-mercaptoethanol, and $0.1 \%$ bromophenol blue at $100^{\circ} \mathrm{C}$ for $5 \mathrm{~min}$. SDS polyacrylamide gels (Bio-Rad laboratories, Hercules, CA, USA) were loaded with $25 \mu \mathrm{g}$ total protein per lane. Prestained protein molecular weight markers (BioRad) were run as standards. The electrophoresed samples were transferred to PVDF membranes (Millipore, Billerica, MA, USA). After transfer, the membrane was blocked with 5\% skim milk in TPBS (200 mM Tris pH 7.0, 1.37 M $\mathrm{NaCl}, 1 \%$ Tween-20) for $1 \mathrm{~h}$ at room temperature. The antiPD-L1 was obtained from Abcam (Cambridge, MA, USA). The antibodies including anti-HER2, anti-phospho-HER2, anti-Akt, anti-phospho-Akt, anti-ERK, anti-phospho-ERK, anti-STAT3, anti-phospho-STAT3, anti-PTEN, and antibeta actin antibodies were purchased from Cell Signaling Technology (Danvers, MA, USA). The membrane was then incubated with diluted primary antibodys for overnight at $4^{\circ} \mathrm{C}$, rinsed and washed, and incubated with the appropriate HRP-conjugated secondary antibody (1:4,000 dilution; Cell Signaling Technology, Danvers, MA, USA) for $2 \mathrm{~h}$ at room temperature. The membrane was washed and rinsed as before, and the expressed proteins were detected with the Pierce ECL Plus Western Blotting Substrate (Amersham Pharmacia Biotech, Buckinghamshire, UK). Each experiment was performed in triplicate.

\section{Flow cytometry analysis}

Cells were centrifuged at $300 \mathrm{~g}$ for $3 \mathrm{~min}$, washed with $\mathrm{PBS}$, and fixed in $70 \%$ ethanol at $-20^{\circ} \mathrm{C}$. After incubation with fluorescently labeled antibodies for $30 \mathrm{~min}$ at $4^{\circ} \mathrm{C}$, cells were washed with PBS buffer and analyzed on a FACSCalibur flow cytometer (Becton Dickinson Biosciences, Heidelberg, Germany) with CellQuest Pro software (10000 events were recorded for each sample).

\section{qPCR and RT-PCR}

Total mRNA was purified from cells using TRIzol reagent (Invitrogen, carsbad, CA, USA) according to the manufacturer's protocol for cultured epithelial cells. The High Capacity RNA-to-cDNA Kit (Applied Biosystems, CA, 
USA) was used to generate cDNA from $1 \mu \mathrm{g}$ of total mRNA. qPCR analysis was performed on the ABI qPCR system (Applied Biosystems) using SYBR Green PCR Master Mix (Applied Biosystems). The RT-PCR amplification program used was $5 \mathrm{~min}$ at $94^{\circ} \mathrm{C}$ followed by 35 cycles at $94^{\circ} \mathrm{C}$ for $30 \mathrm{~s}, 55^{\circ} \mathrm{C}$ for $30 \mathrm{~s}$, and $72^{\circ} \mathrm{C}$ for $30 \mathrm{~s}$. The PCR products were analyzed on $1 \%$ agarose gels (USB Corporation, MA, USA) in 1\% Tris-borate-EDTA buffer stained with DYNE Loading STAR (DYNEBIO, SeongNam, Korea), and they were visualized by a UV transilluminator and recorded using a gel photo imaging system.

The primers used in the PCR reaction were as follows: PD-L1 forward primer 5'-ttgggaaatggaggataaga-3' and reverse primer 5'-ggatgtgccagaggtagttct-3', CCL2 forward primer $5^{\prime}$ - ccccagtcacctgctgttat- $3^{\prime}$ and reverse primer 5'-tggaatcctgaacccacttc-3', CCL21 forward primer $5^{\prime}$-gecttgccacactctttctc-3' and reverse primer 5'-caaggaagaggtggggtgta-3', CXCL1 forward primer $5^{\prime}$-gggaattcaccccaagaac- $3^{\prime}$ and reverse primer $5^{\prime}$-caccagtgagcttcetcctc-3', VEGF forward primer $5^{\prime}$-aaggaggagggcagaatcat-3' and reverse primer 5 '-atctgcatggtgatgttgga- 3 ', and GAPDH forward primer $5^{\prime}$-gcctcaagatcatcagcaatgcet-3' and reverse primer $5^{\prime}$-tgtggtcatgagtccttccacgat- $3^{\prime}$. The relative quantity of mRNA (RQ value) was calculated as $R Q=2^{\Delta \Delta C T}$.

\section{ELISA}

The changes in release of CCL2, CCL21, VEGF and CXCL1 were evaluated using ELISA. Supernatants were collected after $24 \mathrm{~h}$ treatment with lapatinib. The experiment was conducted using individual Human ELISA Kits (Abcam, Cambridge, MA, USA) according to the manufacturer's protocols. The absorbance was read at $450 \mathrm{~nm}$ using a SPECTRA max PLUS 384(Molecular Devices, CA, USA) and data were analyzed with SoftMax Pro software (Molecular Devices, CA, USA).

\section{IHC analysis}

Tissue microarray construction was performed for 251 patients with gastric cancer who underwent gastric resection between 2003 and 2004 at Seoul National University Bundang Hospital, as previously described [17]. Paired specimens were used to observe changes before or after chemotherapy. Commercially available primary antibodies were used according to the manufacturer's instructions (anti-PD-L1 rabbit polyclonal antibody, 1:1500 dilution [Abcam, Cambridge, MA, USA]; anti-HER2/neu rabbit monoclonal antibody [Ventana, AZ, USA]; anti-EGFR mouse monoclonal antibody [Ventana, AZ, USA]). The IHC staining result for PD-L1 was evaluated and categorized as positive when the intensity of PD-L1 was graded as weak, moderate, or strong in $\geq 10 \%$ of the tumor. HER 2 expression was classified as negative, 1+, 2+, or $3+$ as a guideline [18].

\section{Statistical analysis}

All the experiments were repeated three times. The values shown in the tables and figures represent the mean \pm standard deviation of three assays. Associations among groups were evaluated using chi-square test, linear-bylinear association, and one-way analysis of variance (ANOVA). This study for patients' information was approved by the Institutional Review Board of SNUBH (B-1701/378-305). All analyses were performed using Microsoft Excel, SigmaPlot software, and SPSS (SPSS Inc., Chicago, IL, USA).

\section{CONFLICTS OF INTEREST}

All authors declare no conflicts of interest.

\section{FUNDING}

No funding.

\section{REFERENCES}

1. Jain RK. Normalizing tumor microenvironment to treat cancer: bench to bedside to biomarkers. J Clin Oncol. 2013; 31: 2205-2218.

2. Zlotnik A, Yoshie O. The chemokine superfamily revisited. Immunity. 2012; 36:705-716.

3. Freeman GJ, Long AJ, Iwai Y, Bourque K, Chernova T, Nishimura H, Fitz LJ, Malenkovich N, Okazaki T, Byrne MC, Horton HF, Fouser L, Carter L, et al. Engagement of the PD-1 immunoinhibitory receptor by a novel B7 family member leads to negative regulation of lymphocyte activation. J Exp Med. 2000; 192: 1027-1034.

4. Keir ME, Butte MJ, Freeman GJ, Sharpe AH. PD-1 and its ligands in tolerance and immunity. Annu Rev Immunol. 2008; 26: 677-704.

5. Ungefroren H, Sebens S, Seidl D, Lehnert H, Hass R. Interaction of tumor cells with the microenvironment. Cell Commun Signal. 2011; 9: 18.

6. Aldinucci D, Gloghini A, Pinto A, De Filippi R, Carbone A. The classical Hodgkin's lymphoma microenvironment and its role in promoting tumour growth and immune escape. J Pathol. 2010; 221: 248-263.

7. Herbst RS, Soria JC, Kowanetz M, Fine GD, Hamid O, Gordon MS, Sosman JA, McDermott DF, Powderly JD, Gettinger SN, Kohrt HE, Horn L, Lawrence DP, et al. Predictive correlates of response to the anti-PD-L1 antibody MPDL3280A in cancer patients. Nature. 2014; 515: 563-567.

8. Taube JM, Klein A, Brahmer JR, Xu H, Pan X, Kim JH, Chen L, Pardoll DM, Topalian SL, Anders RA. Association of PD-1, PD-1 ligands, and other features of the tumor immune microenvironment with response to anti-PD-1 therapy. Clin Cancer Res. 2014; 20: 5064-5074. 
9. Brahmer J, Reckamp KL, Baas P, Crinò L, Eberhardt WEE, Poddubskaya E, Antonia S, Pluzanski A, Vokes EE, Holgado E, Waterhouse D, Ready N, Gainor J, et al. Nivolumab versus docetaxel in advanced squamous-cell non-small-cell lung cancer. N Engl J Med. 2015; 373: 123-135.

10. D'Incecco A, Andreozzi M, Ludovini V, Rossi E, Capodanno A, Landi L, Tibaldi C, Minuti G, Salvini J, Coppi E, Chella A, Fontanini G, Filice ME, et al. PD-1 and PD-L1 expression in molecularly selected non-small-cell lung cancer patients. Br J Cancer. 2015; 112: 95-102.

11. Azuma K, Ota K, Kawahara A, Hattori S, Iwama E, Harada T, Matsumoto K, Takayama K, Takamori S, Kage M, Hoshino T, Nakanishi Y, Okamoto I. Association of PD-L1 overexpression with activating EGFR mutations in surgically resected nonsmall-cell lung cancer. Ann Oncol. 2014; 25: 1935-1940.

12. Tang Y, Fang W, Zhang Y, Hong S, Kang S, Yan Y, Chen N, Zhan J, He X, Qin T, Li G, Tang W, Peng P, et al. The association between PD-L1 and EGFR status and the prognostic value of PD-L1 in advanced non-small cell lung cancer patients treated with EGFR-TKIs. Oncotarget. 2015; 6: 14209-14219. doi: 10.18632/oncotarget.3694.

13. Loi S, Michiels S, Salgado R, Sirtaine N, Jose V, Fumagalli D, Kellokumpu-Lehtinen PL, Bono P, Kataja V, Desmedt C, Piccart MJ, Loibl S, Denkert C, et al. Tumor infiltrating lymphocytes are prognostic in triple negative breast cancer and predictive for trastuzumab benefit in early breast cancer: results from the FinHER trial. Ann Oncol. 2014; 25: $1544-1550$.

14. Lastwika KJ, Wilson W, Li QK, Norris J, Xu H, Ghazarian SR, Kitagawa H, Kawabata S, Taube JM, Yao S, Liu LN, Gills JJ, Dennis PA. Control of PD-L1 expression by oncogenic activation of the AKT-mTOR pathway in nonsmall cell lung cancer. Cancer Res. 2016; 76: 227-238.

15. Ota K, Azuma K, Kawahara A, Hattori S, Iwama E, Tanizaki J, Harada T, Matsumoto K, Takayama K, Takamori S, Kage M, Hoshino T, Nakanishi Y, et al. Induction of PD-L1 expression by the EML4-ALK oncoprotein and downstream signaling pathways in non-small cell lung cancer. Clin Cancer Res. 2015; 21: 4014-4021.

16. Lin K, Cheng J, Yang T, Li Y, Zhu B. EGFR-TKI downregulates $\mathrm{PD}-\mathrm{L} 1$ in EGFR mutant NSCLC through inhibiting NF-кB. Biochem Biophys Res Commun. 2015; 463: 95-101.

17. Kim JW, Nam KH, Ahn SH, Park DJ, Kim HH, Kim SH, Chang H, Lee JO, Kim YJ, Lee HS, Kim JH, Bang SM, Lee JS, et al. Prognostic implications of immunosuppressive protein expression in tumors as well as immune cell infiltration within the tumor microenvironment in gastric cancer. Gastric Cancer. 2014; 19: 42-52.

18. Bang YJ, Van Cutsem E, Feyereislova A, Chung HC, Shen L, Sawaki A, Lordick F, Ohtsu A, Omuro Y, Satoh T, Aprile G, Kulikov E, Hill J, et al. Trastuzumab in combination with chemotherapy versus chemotherapy alone for treatment of HER2-positive advanced gastric or gastro-oesophageal junction cancer (ToGA): a phase 3, open-label, randomised controlled trial. Lancet. 2010; 376: 687-697.

19. Wimberly H, Brown JR, Schalper K, Haack H, Silver MR, Nixon C, Bossuyt V, Pusztai L, Lannin DR, Rimm DL. PD-L1 expression correlates with tumor-infiltrating lymphocytes and response to neoadjuvant chemotherapy in breast cancer. Cancer Immunol Res. 2015; 3: 326-332.

20. Lin C, Chen X, Li M, Liu J, Qi X, Yang W, Zhang H, Cai Z, Dai Y, Ouyang X. Programmed death-ligand 1 expression predicts tyrosine kinase inhibitor response and better prognosis in a cohort of patients with epidermal growth factor receptor mutation-positive lung adenocarcinoma. Clin Lung Cancer. 2015; 16: e25-35.

21. Han JJ, Kim DW, Koh J, Keam B, Kim TM, Jeon YK, Lee $\mathrm{SH}$, Chung DH, Heo DS. Change in PD-L1 expression after acquiring resistance to gefitinib in EGFR-mutant non-smallcell lung cancer. Clin Lung Cancer. 2016; 17: 263-270.

22. Meniawy TM, Lake RA, McDonnell AM, Millward MJ, Nowak AK. PD-L1 on peripheral blood T lymphocytes is prognostic in patients with non-small cell lung cancer (NSCLC) treated with EGFR inhibitors. Lung Cancer. 2016; 93: 9-16.

23. Böger C, Behrens HM, Mathiak M, Krüger S, Kalthoff H, Röcken C. PD-L1 is an independent prognostic predictor in gastric cancer of Western patients. Oncotarget. 2016; 7: 24269-24283. doi:10.18632/oncotarget.3694.

24. The Cancer Genome Atlas Research Network. Comprehensive molecular characterization of gastric adenocarcinoma. Nature. 2014; 513: 202-209. 